\title{
Localization of fermions in an anisotropic random magnetic field
}

\author{
Yong Baek Kim \\ Department of Physics, The Pennsylvania State University, University Park, PA 16802, U.S.A. \\ Institute for Theoretical Physics, University of California, Santa Barbara, CA 93106, U.S.A.
}

\begin{abstract}
We study the localization of fermions in an anisotropic random magnetic field in two dimensions. It is assumed that the randomness in a particular direction is stronger than those in the other directions. We consider a network model of zero field contours, where there are two types of randomness - the random tunneling matrix element at the saddle points and unidirectional random variation of the number of fermionic states following zero field contours. After averaging over the random complex tunneling amplitude, the problem is mapped to an $S U(2 N)$ random exchange quantum spin chain in the $N \rightarrow 0$ limit. We suggest that the fermionic state becomes critical in an anisotropic fashion.
\end{abstract}

PACS: 72.10.Bg, 72.15.Rn, 71.55.Jv

The localization problem of a nonrelativistic particle in a random perpendicular magnetic field has been a subject of intensive research and debate. In particular, the problem arises from the study of disordered half-filled Landau level in the quantum Hall regime [1]. In the absence of disorder, the Chern-Simons theory of electrons in the half-filled Landau level leads to an effective theory of composite fermions in the fluctuating gauge field [1]. Due to the fact that the density distribution of the fermions are tied to the fluctuating magnetic field, static disorder leads naturally to the random static magnetic field with zero mean [2]. Since the longitudinal resistivity of the electrons in the half-filled Landau level is the same as that of composite fermions, the problem of localization of composite fermions in a random static magnetic field is important for understanding transport properties at the half-filling.

One might expect that the system belongs to the unitary universality class as the disordered system with broken time reversal symmetry and zero Hall conductance. According to conventional scaling theory, all the states are localized in two dimensions for the unitary class [3]. It is established that those systems can be described by a unitary non-linear sigma model $(\mathrm{NL} \sigma \mathrm{M})$ without topological term in the long wavelength limit. Some years ago, Zhang and Arovas [i] suggested that local fluctuation of $\sigma_{x y}$ can induce the long-range interactions between topological densities in the $\mathrm{NL} \sigma \mathrm{M}$. It was claimed that this may give rise to a novel delocalization transition when the conductance at short distances becomes larger than a critical value of the order of $e^{2} / h$.

Since then, it has been controversial whether the model has a delocalized state or not. There have been several numerical and analytical studies leading to different conclusions. Some of them conclude that the system indeed belongs to the unitary class and all the states are localized [5]. On the other hand, some numerical calculations on the lattice model reached the conclusion that there are delocalized states near the band center [4, 8 .
The very recent extensive numerical calculation by $\mathrm{Fu}-$ rusaki [9] showed that a state in the band center is not localized when there is a special particle-hole symmetry in the lattice model. On the other hand, in the absence of the particle-hole symmetry, all the states are localized. This suggests that there is no extended state in the generic continuum model 9 .

Previously Furusaki, Lee, and the author [6] map a network model [5] of snake states (which are fermionic states following zero field contours) to a quantum spin chain. They suggest that the quantum spin chain has a gap in the excitation spectrum so that the fermions in the original problem is always localized. In this paper, we elaborate this mapping further and point out that some ideas of Zhang-Arovas theory may be realized in an anisotropic version of the random flux problem. In particular, we suggest that the number of snake states can fluctuate in space, which was set to be a fixed number in previous numerical and analytical studies. We show that if the number of snake states changes only in a particular direction, the fermionic state becomes critical and the critical behavior could be different for different directions.

Let us first review the mapping from a network model of snake states to a quantum spin chain [6,10]. We assume that the random magnetic field has zero mean and a non-zero variance. We consider the semiclassical limit in which the correlation length of the random magnetic field is large compared to the typical magnetic length. In this limit of smooth disorder, a fermion moves along contours of constant magnetic field. The contours which percolate geometrically across the system would be $B=0$ contours. It turns out that semiclassical motion of a fermion along $B=0$ contours follows snake-like trajectories. In the network model, these zero field contours are represented as links on a square network, carrying the fermionic "snake" states [5]. The node of the network corresponds to the saddle points of the magnetic field where the scattering to either the right or left occurs [5]. The quantum inter- 
ference due to these scattering processes are modeled by random tunneling amplitudes at the saddle points and the mixing of the snake states.

It is important to notice that the fermionic states along the zero field contours arise in pairs at a given energy and they are propagating in the same direction. This is because these states are generated from a symmetric and antisymmetric linear combinations of the Landau levels on either side of the contour. This implies that there exist two snake states per each local Landau level below the Fermi energy. Therefore, there are always even number, $M$, of snake states in each link of the network model. Previous studies assumed that the number of snake states, $M$, is fixed [0.6. However, notice that $M$ could be a spatially varying quantity as far as this is an even number because the magnitude of the magnetic field changes in space. Later, we argue that if the change of $M$ occurs only in one direction, then it leads to a significant effect. At the moment, we assume that $M$ is a fixed even number.

In the network model, two types of random complex tunneling amplitude are introduced, $t_{j}$ and $t_{j k} . t_{j}$ represents the tunneling between the snake states, $\psi_{j}$ $(j=1, \ldots, M)$, in neighboring zero field contours and $t_{j k}$ corresponds to the mixing between snake states in different channels (among $M$ number of channels) in the same zero field contour. Using the replica trick, the disorder averages over $t_{j}$ and $t_{j k}$ are done. Taking $y$ as the imaginary time, one can regard the disorder averaged action as representing interacting fermions, $\psi_{\alpha j}(j=1, \ldots, M$ and $\alpha=1, \ldots, 2 N)$ on $M$ number of coupled one dimensional systems. Here $N$ is the replica index. Substituting the fermion operators with the generators of $S U(2 N)$ :

$$
S_{j, \beta}^{\alpha}=\psi_{\alpha j}^{\dagger} \psi_{\beta j}-\delta_{\alpha \beta} \frac{1}{2 N} \sum_{\gamma} \psi_{\gamma j}^{\dagger} \psi_{\gamma j}
$$

we can obtain $M$ coupled $S U(2 N)$ spin chains given by the Hamiltonian [6]:

$$
\begin{aligned}
H= & \sum_{x} \sum_{j} J_{j} \operatorname{Tr}\left[S_{j}(x+a) S_{j}(x)\right] \\
& +\sum_{x} \sum_{j<k} J_{j k} \operatorname{Tr}\left[S_{j}(x) S_{k}(x)\right],
\end{aligned}
$$

where $a$ is the lattice spacing corresponding to typical distance between snake snakes. Here $J_{j}=\left\langle\left|t_{j}\right|^{2}\right\rangle$, $J_{j k}=-\left\langle\left|t_{j k}\right|^{2}\right\rangle$, and $\operatorname{Tr}[A B]=\sum_{\alpha \beta} A_{\alpha}^{\beta} B_{\beta}^{\alpha}$ for any $2 N \times 2 N$ matrix $A$ and $B$. In the case of decoupled chains, each spin chain is described by a particular representation of $S U(2 N)$, characterized by a Young tableau with a single column of length $N$.

Notice that there are antiferromagnetic couplings $\left(J_{j}>0\right)$ in each spin chain $j$ and ferromagnetic couplings $\left(J_{j k}<0\right)$ between the spin chain $j$ and $k$ for each $x$. The spin at each site represents a snake state at a given time at that particular position. The antiferromagnetic coupling, $J_{j}$, comes from the fact that the tunneling between the snake states in the same channel always occurs between oppositely propagating snake states. On the other hand, the mixing between snake states in different channels happens for snake states propagating in the same direction, which leads to the ferromagnetic coupling, $J_{j k}$. Notice that each chain corresponds to a single-channel network model with non-zero Hall conductance. In order to impose the constraint of zero Hall conductance we require the alternation of the bond strength on the neighboring chains to be staggered. This means that $J_{j}(x=$ even $)=J_{j+1}(x=$ odd $)$ and $J_{j}(x=$ odd $)=J_{j+1}(x=$ even $)$. In terms of the original problem, the localization of the fermions corresponds to the existence of an energy gap in the spin chain (in the replica limit, $N \rightarrow 0$ ).

Due to the fact that there are $M$ number of snake states in each link, the conductance of the system at short distances is $G=M \frac{e^{2}}{h}$. Thus the large conductance limit, $M \gg 2$, corresponds to the large number of coupled chains. The interchain coupling is ferromagnetic and it exists for any two of $M$ spin chains. Thus, as $M$ becomes large we expect that, the coupled chains become essentially single spin chain with a uniform antiferromagnetic coupling $\mathcal{J}$ [6]:

$$
H=\sum_{x} \mathcal{J} \operatorname{Tr}[S(x+a) S(x)]
$$

where $S(x)$ is in the totally symmetric representation described by a Young tableau with $M$ number of columns of length $N$. It is useful to think about the $S U(2)$ case $(N=1)$. In this case, $M$ corresponds to $2 S$, where $S$ is the ordinary spin quantum number. Since $M$ is always an even number, the system can be described by an integer spin chain. Therefore, there is a gap in the excitation spectrum in the spin chain. If one assumes that $N \rightarrow 0$ limit is not singular, this may imply that the electrons are localized in the original problem even for large bare conductances.

Now let us consider the case that the number of snake states is not fixed, but can change in space. In particular, we assume that the number of snake states changes only in one direction. We will discuss the isotropic case later. In this anisotropic random flux problem, we can take the $x$ direction as the direction in which $M$ changes in space. We assume that there are two types of randomness in the problem - one of them is the random tunneling amplitude at the saddle points and the other one is the number of snake states. After performing the average over the random tunneling amplitude, we get

$$
\begin{aligned}
H= & \sum_{p} \sum_{l_{p} \leq x \leq r_{p}} \sum_{p} \sum_{1 \leq j \leq M_{p}} J_{j} \operatorname{Tr}\left[S_{j}(x+a) S_{j}(x)\right] \\
& +\sum_{1 \leq j \leq \operatorname{Min}\left(M_{p}, M_{p+1}\right)} J_{j} \operatorname{Tr}\left[S_{j}\left(l_{p+1}\right) S_{j}\left(r_{p}\right)\right]
\end{aligned}
$$




$$
+\sum_{p} \sum_{l_{p} \leq x \leq r_{p}} \sum_{1 \leq j<k \leq M_{p}} J_{j k} \operatorname{Tr}\left[S_{j}(x) S_{k}(x)\right] .
$$

Here the coupled spin chains consist of many subsystems labeled by $p . l_{p}$ and $r_{p}$ are the positions of the left and right ends of the $p$ th subsystem $\left(l_{p+1}=r_{p}+a\right)$. Each subsystem labeled by $p$ has $M_{p}$ number of coupled spin chains. In general, $M_{p+1}-M_{p}=M_{p}^{\prime}$ is an even number. In the large bare conductance limit, where $M_{p}$ is much larger than 2, the effective Hamiltonian can be written as

$$
\begin{aligned}
H= & \sum_{p} \sum_{l_{p} \leq x \leq r_{p}} \mathcal{J}_{p} \operatorname{Tr}\left[S_{p}(x+a) S_{p}(x)\right] \\
& +\sum_{p} \operatorname{Min}\left(\mathcal{J}_{p}, \mathcal{J}_{p+1}\right) \operatorname{Tr}\left[S_{p+1}\left(l_{p+1}\right) S_{p}\left(r_{p}\right)\right] .
\end{aligned}
$$

Here the system still consists of many subsystems. Each subsystem labeled by $p$ is a uniform antiferromagnetic spin chain in a representation described by a Young tableau with $M_{p}$ number of columns of length $N$.

It is instructive to think about $S U(2)$ case $(N=2)$. In this case, the above Hamiltonian represents the coupled antiferromagnetic integer spin chains. Each integer spin chain has a uniform antiferromagnetic coupling $\mathcal{J}_{p}$ and the spin magnitude $S_{p}=M_{p} / 2$. Thus each subsystem would have a gap in the excitation spectrum if it were an infinite system. Notice that the second term in the Hamiltonian corresponds to the coupling between edge spins of adjacent spin chains. For an integer spin chain with finite size and spin magnitude $S_{p}$, the edge spin has the spin magnitude $S_{p} / 2$ [11]. This can be seen from the Berry phase contribution to the effective action for an integer spin chain of length $L$ :

$$
\begin{aligned}
S_{\mathrm{BP}}= & S_{p} \sum_{x}(-1)^{x / a} \Omega(x) \sim \frac{S_{p}}{2} \int d x \frac{\partial \Omega(x)}{\partial x} \\
= & \frac{S_{p}}{2}[[\Omega(L)-\Omega(0)]+4 \pi Q],
\end{aligned}
$$

where $\Omega(x)$ is the solid angle subtended by the closed path on the surface of a unit sphere defined by the time evolution of a unit vector at $x$. $Q$ is an integer measuring the number of times the spin configuration covers the surface of the sphere. Since $\frac{S_{p}}{2} \times 4 \pi Q=2 \pi Q$, the last term has no effect and can be dropped.

At the interface $x=L$, between the spin chains with $S_{p}$ and $S_{p+1}$, the Berry phase contribution in the limit $L \gg a$ becomes

$$
S_{\mathrm{BP}}=\frac{S_{p}}{2} \Omega(L)-\frac{S_{p+1}}{2} \Omega(L+a) \approx \frac{S_{p}-S_{p+1}}{2} \Omega(L) .
$$

Since $\left(S_{p}-S_{p+1}\right) / 2=\left(M_{p}-M_{p+1}\right) / 4$, the edge spin can be both the half-integer and integer spin. In the system described by Eq.(5), this generates edge spins with random spin magnitude $S_{p}^{\text {edge }}(X)$ at the interface, $X$, between adjacent $p$ th and $(p+1)$ th integer spin chains. Furthermore, depending on whether $r_{p}-l_{p}$ is even or odd, the residual couplings between those edge spins can be either antiferromagnetic or ferromagnetic. The magnitude of the coupling goes as $\mathcal{J}_{p} e^{-\left(r_{p}-l_{p}\right) / \xi_{p}}$, where $\xi_{p}$ is the correlation length. From the above consideration, one can see that the effective coarse-grained Hamiltonian of the system in the case of $S U(2)$ is given by

$$
H=\sum_{X} \mathcal{J}(X) \mathbf{S}^{\text {edge }}(X+A) \cdot \mathbf{S}^{\text {edge }}(X),
$$

where $\mathcal{J}$ has a distribution of both antiferromagnetic $(\mathcal{J}>0)$ and ferromagnetic couplings $(\mathcal{J}<0)$. Here the spin magnitude can be also random in space and $A$ is the typical distance between interfaces of different subchains.

Coming back to $S U(2 N)$ case, one can generalize the above argument. For example, at the interface, $X$, between two adjacent spin chains, the edge spin is generated and it is in the representation characterized by a Young tableau with $M^{\text {edge }}=($ even number $) / 2$ number of columns of length $N$. At the same time, the sign and magnitude of the residual spin exchange interaction between those edge spins can be random. Thus the effective Hamiltonian can be written as

$$
H=\sum_{X} \mathcal{J}(X) \operatorname{Tr}\left[S^{\text {edge }}(X+A) S^{\text {edge }}(X)\right] .
$$

The number of columns of the Young tableau for the edge spins at $X$ has a random distribution.

It is rather easy to understand what the effective Hamiltonian stands for in the original snake-states picture. In the region where the number of snake states changes from one even number to the other, the number of remaining snake states after forming all possible localized configuration out of available snake states, is still always even and they are all in the same direction. Since most of the snake states in the bulk are localized, the remaining degrees of freedom are described by the weak coupling across a long distance between residual snake states at the interface between two region of different number of snake states. The number of residual snake states can be an arbitrary even number. In the limit of strong mixing, the residual snake states can be represented by a single large snake state. In the spin chain language, the residual snake states correspond to $S^{\text {edge }}$. Thus the number of columns in the Young tableau corresponds to the number of residual snake states at the interface. If the weak coupling occurs between the residual snake states propagating in the same direction, the coupling in the spin chain is ferromagnetic. For the snake states propagating in the opposite direction, the coupling becomes antiferromagnetic.

The $S U(2)$ case described by the Hamiltonian Eq. (8) was studied by various methods 12,13]. It turns out 
that the real space renormalization group (RG) 14, 15 treatment can provide a lot of insight about the ground state of the system [12]. Let us consider an isolated link with two sites, where two neighboring spins are coupled by $\mathcal{J}$. The ground state of an isolated two-site system would have maximum $(\mathcal{J}<0)$ or minimum $(\mathcal{J}>0)$ spin quantum number depending on the sign of the exchange coupling. There would be an energy gap $\Delta$ to the first excited state. Since the total system consists of these links, the system can be described by a distribution of energy gaps and the spin magnitude. In the real space RG scheme, one successively replaces the strongest links, where the energy gap is the largest, by effective spins. The RG transformation preserves the form of the Hamiltonian, but it changes the probability distribution of the gaps (or exchange couplings) and the spin magnitude.

In the low energy limit, the RG flows reach a single universal fixed point and the distributions become universal at the fixed point [12]. At low energies, the random spin chain can be described by weakly coupled large effective spins [12]. As the links are replaced by effective spins, the effective couplings or the gaps of the links decrease. At the same time, the average distance between neighboring spins and the magnitude of the effective spins increase. It was found that the average size of the effective spins diverges as $T^{-\alpha}$ while the average distance between neighboring effective spins increases as $T^{-2 \alpha}$ [12]. That is, the correlation length diverges in the zero temperature limit. This suggests that the following correlation function decays as a power law at the zero temperature [12].

$$
C(X-Y)=\left\langle\mathcal{E}_{X Y} \mathbf{S}(X) \cdot \mathbf{S}(Y)\right\rangle \propto \frac{1}{|X-Y|^{\nu}},
$$

where $\mathcal{E}_{X Y}=\Pi_{Z=X}^{Y-1} \operatorname{sgn}(-\mathcal{J}(Z))$.

In the original problem of fermions in an anisotropic random flux distribution, this may imply that the fermionic state is critical in the direction of changing number of snake states or large variation of the magnitude of random magnetic field. This means that the wavefunction is not exponentially localized in this direction, but decays as a power law as a function of system size. Therefore, in some sense, it behaves like a critical state at the transition between metal and insulator. The reason why the correlation becomes critical is that some rare events across the system in the $x$-direction dominates the disorder averaged correlation function. Since the spin chain does not have an excitation gap, the disorder averaged correlation in the time direction cannot decay exponentially. Due to the critical nature of the spin chain, we expect that it decays also as a power law even though the power law would be in general different for different directions. Thus, the fermionic state in the $y$-direction is also critical and the critical behavior may be different from that in the $x$-direction. In the absence of detailed information about the exponents $\nu$ for different directions, it is hard to tell in which direction the wavefunction decays faster.

In the isotropic problem, the number of snake states can change in any direction. In the spin chain language, it will induce the time and space dependent random spin size and the couplings. Thus one gets an annealed disorder problem instead of a quenched one. Even though it is not clear what the effects of this type of disorder would be, it is possible that the effects of annealed disorder could be much weaker so that the random-tunneling-only model may be enough to describe the isotropic random flux problem.

In conclusion, we consider the motion of fermions in an anisotropic random magnetic field. We assume that the number of fermionic states following zero field contours can be changed only in one direction. The mapping from a corresponding network model to a random quantum spin leads to the conclusion that the fermionic states are critical in an anisotropic fashion.

We thank Akira Furusaki, Ilya Gruzberg, Steve Kivelson, T. Senthil and especially Shou-Cheng Zhang for helpful discussions. The research at ITP, UCSB was supported in part by NSF grant No. PHY9407194. This work was also supported by Alfred P. Sloan Foundation Fellowship.

[1] B. I. Halperin, P. A. Lee, and N. Read, Phys. Rev. B 47, 7312 (1993).

[2] V. Kalmeyer and S.-C. Zhang, Phys. Rev. B 46, 9889 (1992).

[3] P. A. Lee and T. V. Ramakrishnan, Rev. Mod. Phys. 57, 287 (1985).

[4] S.-C. Zhang and D. P. Arovas, Phys. Rev. Lett. 72, 1886 (1994).

[5] D. K. K. Lee and J. T. Chalker, Phys. Rev. Lett. 72, 1510 (1994); D. K. K. Lee, J. T. Chalker, and D. Y. K. Ko, Phys. Rev. B 50, 5272 (1994).

[6] Y. B. Kim, A. Furusaki, and D. K. K. Lee, Phys. Rev. B 52, 16646 (1995).

[7] T. Sugiyama and N. Nagaosa, Phys. Rev. Lett. 70, 1980 (1993); A. G. Aronov, A. D. Mirlin, and P. Wölfle, Phys. Rev. B 49, 16609 (1994); Y. Avishai, Y. Hatsugai, and M. Khomoto, Phys. Rev. B 47, 9561 (1993).

[8] D. Z. Liu, X. C. Xie, S. Das Sarma, and S.-C.Zhang, Phys. Rev. B 52, 5858 (1995); D. N. Sheng and Z. Y. Weng, Phys. Rev. Lett. 75, 2388 (1995); J. Miller and J. Wang, Phys. Rev. Lett. 76, 1461 (1996); K. Yang and R. N. Bhatt, Phys. Rev. B 55, 1922 (1997).

[9] A. Furusaki, Phys. Rev. Lett. 82, 604 (1999).

[10] D. H. Lee, Phys. Rev. B 50, 10788 (1994).

[11] T. K. Ng, Phys. Rev. B 47, 11575 (1993).

[12] E. Westerberg, A. Furusaki, M. Sigrist, and P. A. Lee, 
Phys. Rev. Lett. 75, 4302 (1995).

[13] A. Furusaki, M. Sigrist, P. A. Lee, K. Tanaka, and N. Nagaosa, Phys. Rev. Lett. 73, 2622 (1994).
[14] S.-K. Ma, C. Dasgupta, and C.-K. Hu, Phys. Rev. Lett. 43, 1434 (1979).

[15] D. S. Fisher, Phys. Rev. B 50, 3799 (1994). 